\title{
A shear heating origin for ridges on Triton
}

\author{
Louise M. Prockter, ${ }^{1}$ Francis Nimmo, ${ }^{2}$ and Robert T. Pappalardo ${ }^{3}$ \\ Received 25 February 2005; revised 27 May 2005; accepted 9 June 2005; published 30 July 2005.
}

[1] Triton and Europa each display a variety of distinctive curvilinear ridges and associated troughs. Here we propose that ridges on Triton may have formed by diurnal tidal stresses, in a manner similar to that proposed for ridge formation on Europa. The greater width of ridges on Triton is likely a consequence of the lower surface temperature and greater brittle-ductile transition depth on this body compared to Europa. The magnitudes of the stresses and heat fluxes required to generate ridges of the correct scale are comparable to likely values generated during the latter part of Triton's orbital evolution from an initial highly eccentric state. Citation: Prockter, L. M., F. Nimmo, and R. T. Pappalardo (2005), A shear heating origin for ridges on Triton, Geophys. Res. Lett., 32, L14202, doi:10.1029/2005GL022832.

\section{Introduction}

[2] Triton is believed to be a captured satellite based on its retrograde orbit around Neptune. If Triton's capture occurred relatively early in solar system history, it might have been enabled by gas drag; alternatively, more recent capture may have been facilitated by collision with an original Neptunian satellite [Goldreich et al., 1989; Benner and McKinnon, 1995]. Following capture, the orbit is predicted to circularize on a timescale of $\sim 1$ By [Ross and Schubert, 1990]. Both Triton and Europa have relatively young surface ages, $100 \mathrm{Ma}$ and 60 Ma respectively [Stern and McKinnon, 2000; Schenk et al., 2004], as demonstrated by the small number of visible impact craters. Both moons are likely still geologically active [e.g., Kirk et al., 1995; Phillips et al., 2000]. A myriad of exotic ices are observed spectroscopically on Triton [e.g., Quirico et al., 1999], but water ice or ammonia hydrate ice is thought to be the "bedrock ice" in order to preserve topographic relief [Croft et al., 1995; Cruikshank et al., 2000]. Europa's surface is primarily comprised of water ice, along with hydrated contaminants [McCord et al., 1999].

\section{Observations}

[3] Voyager 2 imaged only $20 \%$ of Triton's Neptunefacing hemisphere at resolutions of $1 \mathrm{~km} / \mathrm{pixel}$ or better [Smith et al., 1989]. Galileo imaged about $40 \%$ of Europa's surface at regional resolutions of a few hundred $\mathrm{m} /$ pixel.

\footnotetext{
${ }^{1}$ Planetary Exploration Group, Johns Hopkins University Applied Physics Laboratory, Laurel, Maryland, USA.

${ }^{2}$ Department of Earth Sciences, University of Santa Cruz, Santa Cruz, California, USA.

${ }^{3}$ Laboratory for Atmosphere and Space Physics, University of Colorado, Boulder, Colorado, USA.

Copyright 2005 by the American Geophysical Union. 0094-8276/05/2005GL022832\$05.00
}

Images from both moons show linear ridges with similar morphologies that are not observed on any other planetary bodies (Figure 1). Europan ridges span a range from isolated troughs to complex multi-ridged structures [Head et al., 1999; Figueredo and Greeley, 2000], but the most ubiquitous landform is the double ridge, consisting of a prominent trough flanked by two upraised rims. Complex ridges may contain multiple subparallel ridges, and are less common.

[4] Triton ridges have remarkably similar morphological forms [Smith et al., 1989] to those on Europa, and include single isolated troughs and double ridges, which are apparent especially within Triton's "cantaloupe terrain." Triple and multi-crested ridges are also present, but are rare. In general, Triton's ridges tend to be morphologically subdued compared to Europa's ridges, but are much larger in overall scale.

[5] Despite their similarities, some differences in ridge morphology do exist. Margins of Triton ridges are less distinct than those on Europa, and their termini show greater morphological variation. It is commonly difficult to determine superposition of features on Triton (plausibly due to limits of image resolution), but there are some instances where younger ridges clearly overlie older ridges.

[6] Profiles were obtained from stereo imaging across a typical double ridge in the anti-Jovian region of Europa [Giese et al., 1999; Nimmo et al., 2003], and composite topographic profiles across a ridge on Triton were produced by Croft et al. [1995] based on 2-D photoclinometry (Figure 2). Despite differences in horizontal scale, the topographic profiles across ridges on Europa and Triton both clearly show well-pronounced central troughs and flanking ridge crests.

[7] Galileo images of Europa have shown bounding depressions to be common alongside ridges, and they are interpreted to be flexural features [Head et al., 1999]. Topographic profiles constructed for two of Triton's ridges (Figure 2) show clear depressions flanking the ridge. Such depressions on Triton have been suggested to be graben, into which ridges were cryovolcanically extruded [Smith et al., 1989]. Several models have been proposed for the formation of Europan ridges, each of which has its strengths and weaknesses (see Pappalardo et al. [1999] for comprehensive review). We here focus on the shear heating model of Gaidos and Nimmo [2000] and Nimmo and Gaidos [2002] in which heating along cracks from diurnal tidally driven strike-slip motion causes upwelling of warm ice to form ridges, with possible associated partial melting. The shear heating model is consistent with the strike-slip offsets noted along many, if not most Europa ridges [Hoppa et al., 1999a], and does not require the presence of liquid water close to the surface. Below we argue that the shear heating model may also explain ridge formation on Triton, as a 


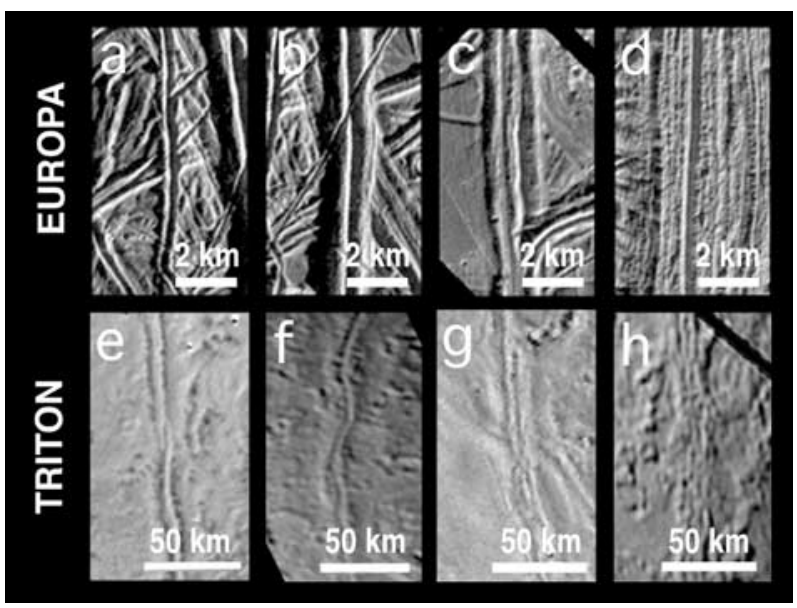

Figure 1. Comparison of Europa and Triton ridge morphology: (a and e) Raised flank trough; ( $b$ and $\mathrm{f}$ ) Double ridge; (c and g) Triple ridge; ( $\mathrm{d}$ and $\mathrm{h}$ ) Complex ridge.

result of the high transient diurnal stresses this body experienced early in its history.

\section{Modeling}

\subsection{Stresses}

[8] Following capture into a highly eccentric orbit, tidal dissipation would have reduced Triton's semi-major axis $a$, and eccentricity $e$, with a timescale dependent on the dissipation factor $Q$ and Love number $k$ of the satellite. In modeling the circularization of Triton's retrograde orbit, Ross and Schubert [1990] show that there is a peak in tidal dissipation during a brief period when the semi-major axis is reduced but eccentricity remains relatively high.

[9] Employing the stress model of Stempel et al. [2004], we determine diurnal stresses on Triton through time, based on the coupled evolution of $a$ and $e$ after its capture as modeled by Ross and Schubert [1990]. The model assumes an ice shell that deforms above a global liquid ocean, which is likely considering the great amount of tidal dissipation following capture by Neptune [McKinnon et al., 1995]. The model neglects higher order terms in $e$, which means that the stresses calculated are underestimates for the likely

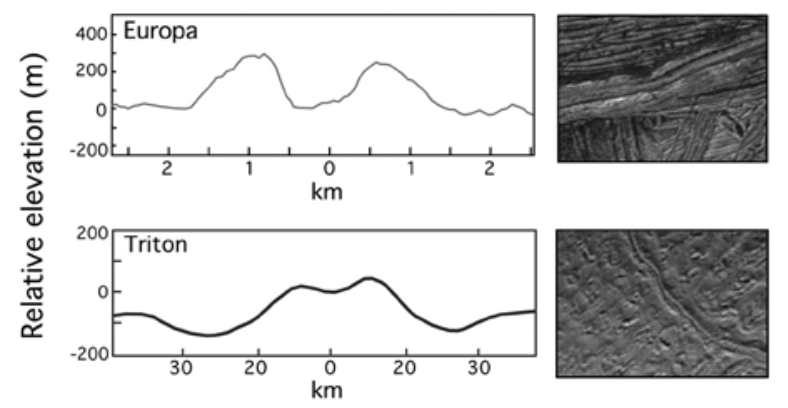

Figure 2. Topographic ridge profiles. (top) After Nimmo et al. [2003, Figure 1] and Giese et al. [1999]. (bottom) From Croft et al. [1995, Figure 19]. Despite differences in scale, ridges on Europa and Triton are topographically similar. stresses on Triton during its period of highest eccentricity. We also assume that $k / Q$, which determines the orbital evolution timescale, is a constant value appropriate to the later stages of Triton's evolution.

[10] Figure 3 shows the evolution of both tidal heating and maximum equatorial diurnal tidal stresses, which peak at $\sim 2 \mathrm{MPa}$ when $e=0.36$. Importantly, this peak in stresses occurs subsequent to the peak in tidal dissipation. We have chosen a relatively small value of $k / Q$, such that by the time the stresses have dropped to $0.5 \mathrm{MPa}$ (at $e=0.06$ ), the heat flux has diminished to $20 \mathrm{~mW} \mathrm{~m} \mathrm{~m}^{-2}$, implying an equilibrium conductive ice shell thickness $\approx 35 \mathrm{~km}$. Thus, for the case presented, the stresses remain high (in fact, an order of magnitude larger than the diurnal stresses on Europa) despite the presence of a relatively thick brittle ice layer. It is this combination of high stresses and brittle ice thickness which we argue is responsible for double ridge formation.

\subsection{Modeling of Ridge Formation}

[11] Diurnal stresses can lead to cyclical shear motion [Hoppa et al., 2001], which in turn may generate shear heating along a fracture plane. Shear heating on Europa may be responsible for the development of double ridges [Gaidos and Nimmo, 2000; Nimmo and Gaidos, 2002], and here we apply a slightly modified version of the shear heating model to the case of Triton.

[12] The model consists of a two-dimensional rectangular section perpendicular to the direction of shear motion, and is described in more detail in Nimmo and Gaidos [2002]. Shear motion is confined to a single vertical fault surface at shallow depths, but at greater depths is accommodated by distributed flow of viscous ice across a broader zone. The shear velocity is specified at the vertical boundaries of the

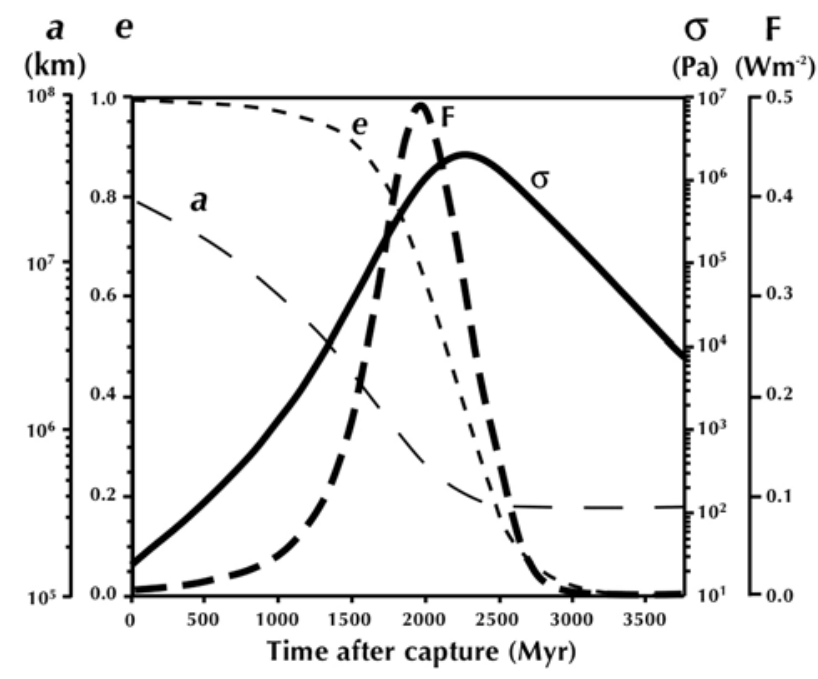

Figure 3. Plot showing evolution of Triton's eccentricity $(e)$, semi-major axis $(a)$, diurnal stress $(\sigma)$ and surface heat flux $(F)$ with time [cf. Ross and Schubert, 1990, Figure 1]; we use a mass ratio of $2.1 \times 10^{-4}, k / Q=3.3 \times 10^{-4}$, and Love numbers $k=0.207$ and $h=0.916$. The evolution timescale and maximum heat flux are determined by $k / Q$ (assumed constant); note that the peak in stress always postdates the peak in tidal heating. 

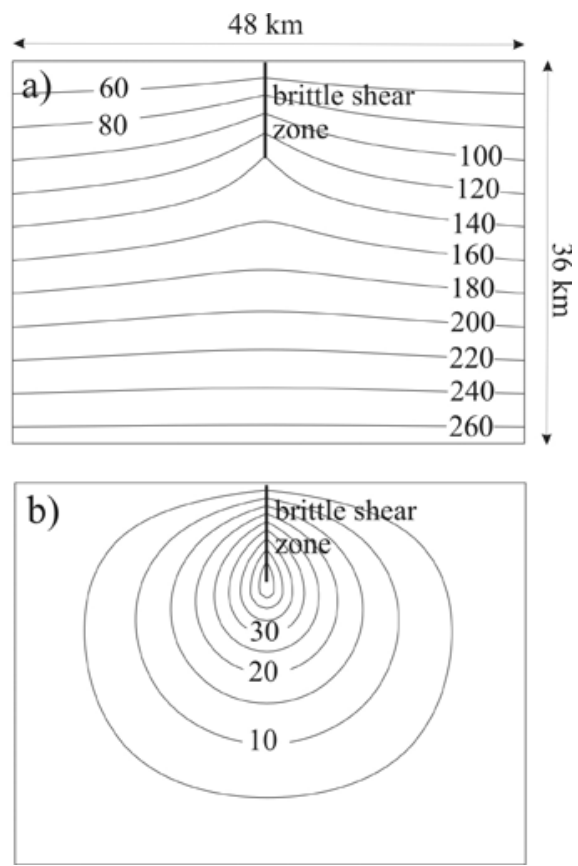

Figure 4. Model of shear heating with self-consistent calculation of brittle-ductile transition (BDT) depth. (a) Temperature structure, contour interval is $20 \mathrm{~K}$, horizontal width $48 \mathrm{~km}$, vertical extent $36 \mathrm{~km}$; surface temperature $40 \mathrm{~K}$, base temperature $270 \mathrm{~K}$, and there are 71 nodes both vertically and horizontally. BDT depth is $9.5 \mathrm{~km}$. Reference viscosity $10^{14} \mathrm{~Pa} \mathrm{~s}$, shear velocity $3 \times 10^{-8} \mathrm{~m} \mathrm{~s}^{-1}$, gravity $0.78 \mathrm{~m} \mathrm{~s}^{-2}$, and other parameters identical to Nimmo and Gaidos [2002, Table 1]. (b) As for Figure 4a, but showing temperature in excess of background conductive temperature structure. Contour interval is $5 \mathrm{~K}$.

section; the cyclic nature of the velocity is not modeled because the orbital period of Triton is short (5.88 days) compared to the thermal diffusion timescale. Temperatures are fixed at the top and bottom boundaries and advection of heat is neglected. Friction on the fault surface and viscous dissipation within the ice both lead to heating which modifies the viscosity structure, which in turn affects the horizontal velocity profile. The model updates the coupled temperature and velocity fields using a finite-difference technique on a rectangular grid until both are in steady state.

[13] In the original model of Nimmo and Gaidos [2002], the thickness of the near-surface brittle faulted layer was kept constant. In reality, however, the thickness of this layer depends on the relative magnitudes of viscous and frictional stresses, and may thus evolve with time as the temperature distribution evolves. Here we calculate the potential frictional and viscous stresses at each node on the fault surface, and hence determine the location of the brittle-ductile transition (BDT) at each timestep. Calculating the BDT depth in a self-consistent manner is important because it controls the width of the features produced.

[14] Figure 4 shows the results of a typical model, based on the plausible late-stage stresses and heat fluxes of Figure 3. Note that this is intended to be a representative example demonstrating that shear-heating is a viable mechanism; given the very large uncertainties in important parameters, the exact values used should not be taken too literally. Figure $4 \mathrm{a}$ shows the steady-state temperature field for a shear velocity of $3 \times 10^{-8} \mathrm{~m} \mathrm{~s}^{-1}$. The self-consistent BDT depth is $9.5 \mathrm{~km}$ and occurs at $146 \mathrm{~K}$. By contrast, on Europa the BDT is estimated at $1-3 \mathrm{~km}$, plausibly accounting for the difference in ridge scale between the two moons.

[15] Figure $4 \mathrm{~b}$ shows the temperature in excess of the background temperature, and demonstrates that the maximum temperature rise $(45 \mathrm{~K})$ is at the base of the brittle zone, as expected. The maximum stress at the base of the brittle zone is $0.75 \mathrm{MPa}$ (for a friction coefficient of 0.1 ). Significantly, this stress is consistent with the maximum stresses derived from the orbital evolution calculations (see Figure 3) and implies that the shear heating model is plausible. The vertical topography which would be generated by the temperature anomaly shown in Figure $4 \mathrm{~b}$ has an amplitude of $\sim 100 \mathrm{~m}$ and a half-width of $20 \mathrm{~km}$, comparable to the Triton ridges in Figure 2. We note that the temperature-related topography will decay rapidly once shear-heating ceases. However, the elevated temperatures are likely to lead to solid-state flow and diapirism [Gaidos and Nimmo, 2000; Nimmo and Gaidos, 2002], and such effects can generate long-lived topography.

\section{Discussion}

[16] Despite different planetary parameters both Triton and Europa show similar morphological ridges, which may have arisen from diurnal stresses that induced shear heating along fractures. The greater scale of the ridges on Triton is mainly a consequence of the lower surface temperature and thicker ice shell. It is reasonable that double ridges are preferentially located within Triton's cantaloupe terrain, because that region is believed to be associated with vigorous vertical (diapiric) overturn of mobile crustal materials [Schenk and Jackson, 1993]. Depressions alongside major ridges may have formed when buoyant material was depleted from these regions by flow laterally toward the ridge axis, analogous to rim synclines of terrestrial diapirs [e.g., Trusheim, 1960]. Ridges on Triton are much less abundant than on Europa, perhaps because fractures through Triton's thicker lithosphere relieve stress over a greater area. Outside of the cantaloupe region, frosts and icy volcanic deposits appear to overprint older terrains, presumably hiding ridges and troughs [Croft et al., 1995].

[17] As previously demonstrated for Europa [Hoppa et al., 1999b], we find that diurnal stressing is a plausible deformation mechanism on Triton. Significant effects from diurnal stresses are expected only for a satellite with an elliptical orbit, periapse close to its primary planet, and an ice shell that is decoupled from the interior by a global ocean. This means that in our solar system, the icy satellites that are the most plausible candidates for diurnally induced deformation are Europa, Triton, and perhaps Charon (depending upon its early eccentricity about Pluto [McKinnon et al., 1997]). Io is plausibly an additional candidate for significant diurnal stress [Bart et al., 2004] if its rocky lithosphere is able to deform above a magma ocean.

[18] Attempts to compare the pattern of Triton's ridges and troughs to plausible global stress models including despinning and orbital precession have yielded uncertain 
results [Collins and Schenk, 1994; Croft et al., 1995]. Our preliminary comparisons of nonsynchronous rotation stress patterns yield a poor fit to Triton's structural pattern. Polar wander provides an additional possible stress mechanism and global pattern [Leith and McKinnon, 1996; Rubincam, 2003]; ice shell thickening [Nimmo, 2004] would add an isotropic extensional stress component. A valuable test of the diurnal stressing model for Triton would be to compare the pattern of maximum diurnal stresses during the course of an elliptical orbit to the pattern of Triton's structures. However, such a comparison requires inclusion of higher order eccentricity terms in modeling surface stress patterns on Triton, a numerically complex task that has not yet been performed. Furthermore, it might be expected that if diurnal stresses dominated the formation of cracks on Triton, cycloidal structures might result, as is the case on Europa [Hoppa et al., 1999b], however Triton's ridges are significantly more linear. We expect that a larger magnitude of strike-slip motion on Triton might result in more linear fractures, but verification of this hypothesis is also the subject of future work.

[19] The amount of heat generated within Triton after its capture could have easily internally melted the satellite [McKinnon et al., 1995]. When tidal dissipation neared its maximum, endogenic activity probably overturned the satellite's surface rapidly and thoroughly. Because the stress maximum occurs after the dissipation maximum, the surface we see today, including its ridges and troughs, is likely a relic of Triton's waning geological activity. Triton's ridges themselves are not necessarily young, but the young surface age and lack of impact craters implies that they formed relatively recently. Because the timescale for Triton's orbital evolution depends on the poorly known (and time-variable) value of $k / Q$, the time of Triton's capture is not well constrained.

[20] Acknowledgments. We thank McCall Mullen and Kris Spinden for their help on this project, and Geoff Collins for a thoughtful and very useful review. This work was supported in part by NASA's PGG program, and by an Applied Physics Laboratory Janney Fellowship to LP.

\section{References}

Bart, G. D., et al. (2004), Ridges and tidal stress on Io, Icarus, 169, $111-$ 126.

Benner, L., and W. B. McKinnon (1995), Orbital evolution of captured satellites: The effect of solar gravity on Triton's post capture orbit, Icarus, $114,1-20$

Collins, G. C., and P. Schenk (1994), Triton's lineaments: Complex morphology and stress patterns, Proc. Lunar Planet. Sci. Conf. 25th, 277.

Croft, S. K., et al. (1995), Geology of Triton, in Neptune and Triton, edited by D. P. Cruikshank and M. S. Matthews, pp. 879-948, Univ. of Ariz. Press, Tucson.

Cruikshank, D. P., et al. (2000), Water ice on Triton, Icarus, 147, 309-316.

Figueredo, P. H., and R. Greeley (2000), Geologic mapping of the northern leading hemisphere of Europa from Galileo solid-state imaging data, J. Geophys. Res., 105, 22,629-22,646.

Gaidos, E., and F. Nimmo (2000), Tectonics and water on Europa, Nature, $405,637$.

Giese, B., et al. (1999), Doublet ridge formation on Europa: Evidence from topographic data, paper presented at Annual Meeting of the Division for Planetary Sciences, Am. Astron. Soc., Padua, Italy.
Goldreich, P., et al. (1989), Neptune's story, Science, 245, 500.

Head, J. W., R. T. Pappalardo, and R. S. Sullivan (1999), Europa: Morphological characteristics of ridges and triple bands from Galileo data (E4 and E6) and assessment of a linear diapirism model, J. Geophys. Res., $104,24,223-24,236$.

Hoppa, G. V., et al. (1999a), Strike slip faults on Europa: Global shear patterns driven by tidal stress, Icarus, 141, 287-298.

Hoppa, G. V., et al. (1999b), Formation of cycloidal features on Europa, Science, 285, 1899-1902.

Hoppa, G. V., et al. (2001), Europa's rate of rotation derived from the tectonic sequence in the Astypalaea region, Icarus, 153, 208-213.

Kirk, R. L., et al. (1995), Triton's plumes: Discovery, characteristics, and models, in Neptune and Triton, edited by D. P. Cruikshank and M. S. Matthews, pp. 949-990, Univ. of Ariz. Press, Tucson.

Leith, A. C., and W. B. McKinnon (1996), Is there evidence for polar wander on Europa?, Icarus, 120, 387-398.

McCord, T. B., et al. (1999), Hydrated salt minerals on Europa's surface from the Galileo Near-Infrared Mapping Spectrometer (NIMS) investigation, J. Geophys. Res., 104, 11,827-11,852.

McKinnon, W. B., J. I. Lunine, and D. Banfield (1995), Origin and evolution of Triton, in Neptune and Triton, edited by D. P. Cruikshank and M. S. Matthews, pp. 807-877, Univ. of Ariz. Press, Tucson.

McKinnon, W. B., D. P. Simonelli, and G. Schubert (1997), Composition, internal structure, and thermal evolution of Pluto and Charon, in Pluto and Charon, edited by S. A. Stern and D. J. Tholen, pp. 295-343, Univ. of Ariz. Press, Tucson.

Nimmo, F. (2004), Stresses generated in cooling viscoelastic ice shells: Application to Europa, J. Geophys. Res., 109, E12001, doi:10.1029/ 2004JE002347.

Nimmo, F., and E. Gaidos (2002), Strike-slip motion and double ridge formation on Europa, J. Geophys. Res., 107(E4), 5021, doi:10.1029/ 2000JE001476.

Nimmo, F., R. T. Pappalardo, and B. Giese (2003), On the origins of band topography, Europa, Icarus, 166, 21-32.

Pappalardo, R. T., et al. (1999), Does Europa have a subsurface ocean? Evaluation of the geological evidence, J. Geophys. Res., 104, 24,015$24,056$.

Phillips, C. B., et al. (2000), The search for geological activity on Europa, J. Geophys. Res., 105, 22,579-22,597.

Quirico, E., et al. (1999), Composition, physical state, and distribution of ices at the surface of Triton, Icarus, 139, 159-178.

Ross, M. N., and G. Schubert (1990), The coupled orbital and thermal evolution of Triton, Geophys. Res. Lett., 17, 1749-1752.

Rubincam, D. P. (2003), Polar wander on Triton and Pluto due to volatile migration, Icarus, 163, 469-478.

Schenk, P. M., and M. P. A. Jackson (1993), Diapirism on Triton: A record of crustal layering and instability, Geology, 21, 299-302.

Schenk, P. M., et al. (2004), Ages and interiors: The cratering record of the Galilean satellites, in Jupiter: The Planet, Satellites and Magnetosphere, edited by F. Bagenal, pp. 427-456, Cambridge Univ. Press, New York.

Smith, B. A., et al. (1989), Voyager 2 at Neptune: Imaging science results, Science, 246, 1422-1449.

Stempel, M., et al. (2004), Combined effects of diurnal and nonsynchronous surface stresses on Europa, Proc. Lunar. Planet. Sci. Conf., [CD-ROM], $35,2061$.

Stern, S. A., and W. B. McKinnon (2000), Triton's surface age and impactor population revisited in light of Kuiper Belt fluxes: Evidence for small Kuiper Belt objects and recent geological activity, Astron. J., $119,945-952$.

Trusheim, F. (1960), Mechanism of salt migration in northern Germany, Assoc. Pet. Geol. Bull., 44, 1519-1540.

F. Nimmo, Department of Earth Sciences, University of Santa Cruz, Santa Cruz, CA 95064, USA.

R. T. Pappalardo, Laboratory for Atmosphere and Space Physics, University of Colorado, Boulder, CO 80309, USA.

L. M. Prockter, Planetary Exploration Group, Johns Hopkins University Applied Physics Laboratory, MP3-E128, 11100 Johns Hopkins Road, Laurel, MD 20723, USA. (louise.prockter@jhuapl.edu) 